Article

\title{
Variations in the Volatile Compositions of Curcuma Species
}

\author{
Noura S. Dosoky ${ }^{1}$, Prabodh Satyal ${ }^{2}$ and William N. Setzer $1,2, *$ (i) \\ 1 Aromatic Plant Research Center, 230 N 1200 E, Suite 100, Lehi, UT 84043, USA; ndosoky@aromaticplant.org \\ 2 Department of Chemistry, University of Alabama in Huntsville, Huntsville, AL 35899, USA; \\ psatyal@aromaticplant.org \\ * Correspondence: wsetzer@chemistry.uah.edu; Tel.: +1-256-824-6519
}

Received: 12 January 2019; Accepted: 30 January 2019; Published: 2 February 2019

check for

updates

\begin{abstract}
Curcuma species have been cultivated in tropical and subtropical regions in Asia, Australia, and South America for culinary as well as medicinal applications. The biological activities of Curcuma have been attributed to the non-volatile curcuminoids as well as to volatile terpenoids. Curcuma essential oils have demonstrated a wide variety of pharmacological properties. The objective of this work was to examine the variation in the compositions of Curcuma rhizome essential oils. In this work, the volatile oils from C. longa and C. zedoaria were obtained and analyzed by gas chromatography-mass spectrometry. The chemical compositions of C. longa and C. zedoaria essential oils, including those reported in the literature, were analyzed by hierarchical cluster analysis. In addition, cluster analyses of the chemical compositions of $C$. aromatica and C. aeruginosa from the literature were also carried out. Curcuma longa volatiles were dominated by $\alpha$-turmerone, curlone, ar-turmerone, $\beta$-sesquiphellandrene, $\alpha$-zingiberene, germacrone, terpinolene, ar-curcumene, and $\alpha$-phellandrene and showed four distinct chemical clusters. C. zedoaria rhizome oil contained 1,8-cineole, curzerenone/epi-curzerenone, $\alpha$-copaene, camphor, $\beta$-caryophyllene, elemol, germacrone, curzerene, and $\beta$-elemene and showed two different chemical types. C. aromatica had three clearly defined clusters, and C. aeruginosa had three types.
\end{abstract}

Keywords: Curcuma aeruginosa; Curcuma longa; Curcuma zedoaria; Curcuma aromatica; rhizome essential oils

\section{Introduction}

The genus Curcuma L. (Zingiberaceae) consists of about 93-100 species of perennial rhizomatous herbs that originated in tropical and subtropical regions of Asia, Australia, and South America [1]. Many of these species are extensively grown on a very large scale in India, Pakistan, Indonesia, Malaysia, Bangladesh, Nepal, and Thailand [2]. Curcuma species are greatly valued for their medicinal properties. For hundreds of years, members of Curcuma have been used in traditional medicine for treating respiratory complaints, pain, digestive disorders, inflammatory conditions, wounds, hypercholesterolemia, hypertension, hematologic and circulation abnormalities, infectious diseases, and cancer prevention, among others [3-5]. They are also important sources of flavoring and coloring agents, cosmetics, perfumes, and ornamental plants [5,6]. Curcuma species possess a variety of pharmacological activities including anti-inflammatory, antiproliferative, anticancer, hypoglycemic, anti-hyperlipidemic, antiatherosclerotic, neuroprotective, hepatoprotective, anti-diarrheal, carminative, diuretic, antirheumatic, anticonvulsant, hypotensive, antioxidant, insecticidal, larvicidal, antimicrobial, antiviral, antivenomous, anti-thrombotic, and antityrosinase activities [7-15].

The rhizome, which contains a variety of terpenoids, flavonoids, and phenylpropanoids [16], is the most extensively used part of the plant [17]. Several studies indicated that the bioactive ingredients 
of Curcuma rhizome are the non-volatile curcuminoids (curcumin, demethoxycurcumin, and bisdemethoxycurcumin) and the volatile oil (sesquiterpenoids and monoterpenoids) [14,18]. Curcumin, the most active curcuminoid in turmeric rhizome, has anticancer [19], anti-inflammatory [20], antioxidant [21], antibacterial, anti-fungal [22], analgesic, digestive, antidepressant [23], and hypoglycemic [23] properties and has shown potential against cardiovascular diseases [24] and Alzheimer's disease [25]. Curcuma essential oil (EO) is often extracted by distillation of the fresh or dry rhizome [26], or by supercritical fluid extraction [27]. Generally, the Curcuma oils are made up of sesquiterpenoids and monoterpenoids [5]. There is a great variation in the literature on Curcuma EO due to differences in the genotype, edaphic factors, climate, time of harvest, extraction, and analysis methods [28-30]. Around 31 Curcuma species have been studied of which C. longa (turmeric) and C. zedoaria (zedoary) are the most extensively investigated [5]. The current study was conducted to investigate the composition and different chemotypes of the rhizome essential oils of C. longa L., C. aromatica Salisb., C. zedoaria (Christm.) Roscoe, and C. aeruginosa Roxb. from collections from different geographical origins.

\section{Materials and Methods}

\subsection{Volatile Oils}

Volatile oils from commercial suppliers were obtained from the collections of the Aromatic Plant Research Center (APRC, Lehi, UT, USA). A total of 33 Curcuma longa (turmeric) rhizome oils from the APRC collection, including 24 hydro- or steam-distilled essential oils, five supercritical $\mathrm{CO}_{2}$ extracts, and four oils of unknown origin or extraction method, were analyzed by gas chromatography-mass spectrometry (GC-MS).

\subsection{Gas Chromatographic-Mass Spectral Analysis}

The essential oils obtained from APRC were analyzed by gas chromatography-mass spectrometry (GC-MS) using a Shimadzu GCMS-QP2010 Ultra operated in the electron impact (EI) mode (electron energy $=70 \mathrm{eV})$, scan range $=40-400$ atomic mass units, scan rate $=3.0 \mathrm{scans} / \mathrm{s}$, and GC-MS solution software (Shimadzu Scientific Instruments, Columbia, MD, USA). The GC column was a ZB-5 fused silica capillary column with a (5\% phenyl)-polymethylsiloxane stationary phase and a film thickness of $0.25 \mu \mathrm{m}$, a length of $30 \mathrm{~m}$, and an internal diameter of $0.25 \mathrm{~mm}$ (Phenomenex, Torrance, CA, USA). The carrier gas was helium with a column head pressure of $552 \mathrm{kPa}$ and flow rate of $1.37 \mathrm{~mL} / \mathrm{min}$. The injector temperature was $250{ }^{\circ} \mathrm{C}$ and the ion source temperature was $200{ }^{\circ} \mathrm{C}$. The GC oven temperature was programmed for $50^{\circ} \mathrm{C}$ initial temperature, then temperature was increased at a rate of $2{ }^{\circ} \mathrm{C} / \mathrm{min}$ to $260^{\circ} \mathrm{C}$. A $7 \% w / v$ solution of the sample was prepared in dichloromethane and $0.1 \mu \mathrm{L}$ was injected with a splitting mode (30:1). Identification of the oil components was based on their retention indices determined by reference to a homologous series of $n$-alkanes, and by comparison of their mass spectral fragmentation patterns with those reported in the literature [31] and our own in-house library [32].

\subsection{Hierarchical Cluster Analysis}

The chemical compositions of the Curcuma oils obtained from this work as well as the published literature were used in the cluster analysis. The essential oil compositions were treated as operational taxonomic units (OTUs), and the concentrations (percentages) of the major components (C. longa: $\alpha$-phellandrene, $p$-cymene, 1,8-cineole, terpinolene, ar-curcumene, $\alpha$-zingiberene, $\beta$-bisabolene, $\beta$-sesquiphellandrene, ar-turmerone (= dehydroturmerone), $\alpha$-turmerone, germacrone, curlone (= $\beta$-turmerone), $(6 S, 7 R)$-bisabolone, and $(E)$ - $\alpha$-atlantone; $C$. zedoaria: 1,8 -cineole, camphor, $\alpha$-copaene, $\beta$-elemene, $\beta$-caryophyllene, ar-curcumene, zingiberene, curzerene, germacrene $\mathrm{B}, \beta$-sesquiphellandrene, curzerenone/epi-curzerenone, and germacrone; $C$. aromatica: $\alpha$-pinene, camphene, 1,8-cineole, camphor, isoborneol, borneol, $\beta$-elemene, ar-curcumene, curzerene, 
$\beta$-curcumene, curzerenone, germacrone, xanthorrhizol, and curdione $(=1$ (10)-germacrene-5,8-dione; C. aeruginosa: camphene, $\beta$-pinene, 1,8-cineole, camphor, isoborneol, borneol, $\beta$-elemene, $\beta$-farnesene, zingiberene, curzerene, germacrene $\mathrm{B}$, curzerenone, $\beta$-eudesmol, germacrone, and curcumenol) were used to determine the chemical associations between the essential oils using agglomerative hierarchical cluster (AHC) analysis using XLSTAT Premium, version 2018.5.53172 (Addinsoft, Paris, France). Dissimilarity was determined using Euclidean distance, and clustering was defined using Ward's method.

\section{Results and Discussion}

Essential oils from the Curcuma species were obtained from a collection of oils from commercial sources deposited with the Aromatic Plant Research Center (APRC). Curcuma species are known for producing an array of volatile sesquiterpenes, monoterpenes, and other aromatic compounds $[5,15]$. Hundreds of compounds have been identified from the turmeric oils, however, the major components were $\alpha$-turmerone (12.6-44.5\%), curlone (9.1-37.8\%), ar-turmerone (12.2-36.6\%), $\beta$-sesquiphellandrene (5.0-14.6\%), $\alpha$-zingiberene (5.0-12.8\%), germacrone (10.3-11.1\%), terpinolene (10.0-10.2\%), ar-curcumene (5.5-9.8\%), and $\alpha$-phellandrene (5.0-6.7\%) (Table 1). Interestingly, Brazilian turmeric EO samples showed (Z)- $\gamma$-atlantone, ar-turmerone, and $(E)$ - $\gamma$-atlantone as the main constituents [33], while a sample from north central Nigeria had $\beta$-bisabolene, $(E)$ - $\beta$-ocimene, $\beta$-myrcene, 1,8-cineole, $\alpha$-thujene, $\alpha$-phellandrene, limonene, zingiberene, and $\beta$-sesquiphellandrene [34]. Turmeric oils of Sri Lanka and São Tomé e Principe origins had $\alpha$-phellandrene, $\alpha$-turmerone, 1,8-cineole, $p$-cymene, ar-turmerone, $\beta$-turmerone, and terpinolene as the major components $[10,35]$.

The rhizome of Curcuma aromatica (commonly known as wild turmeric) is a traditional medicine used to alleviate pain, eliminate blood stasis, and slow ageing [36]. The Japanese $C$. aromatica oil was reported to have curdione (32.2-44.0\%), 1,8-cineole (7.5-25.3\%), and germacrone (4.6-9.6\%) [37], while a sample from Thailand contained camphor $(26.9 \%)$, ar-curcumene $(23.2 \%)$, and xanthorrhizol $(18.7 \%)$ as the main components [38]. Indian samples of $C$. aromatica had camphor (18.2-48.3\%), $\beta$-curcumene (28.4-31.4\%), ar-curcumene (22.1-24.1\%), xanthorrhizol (4.8-16.2\%), 1,8-cineole (5.5-15.9\%), isoborneol $(8.2-12.2 \%)$, curzerenone $(5.5-11.0 \%)$, germacrone $(4.9-10.6 \%)$, camphene $(7.4-10.2 \%)$, curdione $(4.8-8.0 \%)$, borneol $(4.9-8.2 \%), \beta$-elemene $(7.5 \%)$, curzerene $(4.6-6.0 \%), \alpha$-pinene $(5.7-5.9 \%)$, and terpinolene (5.2\%) $[15,37,39-42]$ (Table 2).

Zedoary (Curcuma zedoaria) rhizome is also called "white turmeric" because of its similarity to ginger from the outside and to turmeric from the inside. Zedoary EO is generally made of sesquiterpenoids (80-85\%) and monoterpenoids (15-20\%). The major components of C. zedoaria rhizome oil are 1,8-cineole (7.0-38.4\%), curzerenone / epi-curzerenone (20.9-29.4\%), $\alpha$-copaene $(17.4 \%)$, camphor (8.6-8.8\%), $\beta$-caryophyllene (8.8\%), elemol (6.8\%), germacrone $(6.7 \%)$, curzerene $(5.9 \%)$, and $\beta$-elemene (5.5\%) (Table 3). The main components of $C$. zedoaria rhizome oil reported in the literature were curzerenone/epi-curzerenone (19.0-31.6\%), curzerene (8.0\%), ar-curcumene (12.1\%), zingiberene $(12.0 \%)$, germacrone $(10.8 \%)$, camphor $(10.3 \%), \beta$-sesquiphellandrene $(9.8 \%)$, and germacrene $\mathrm{B}$ $(6.0 \%)[15,43]$.

Curcuma aeruginosa (also known as "black curcuma") is characterized by its distinctive ginger-like scent [44]. The volatile oil of $C$. aeruginosa is known to contain relatively equal amounts of monoterpenes and sesquiterpenes. Two black turmeric samples from Malaysia had curzerenone (24.6-30.4\%), 1,8-cineole (11.2-25.2\%), camphor (6.8-10.5\%), and curcumenol (5.6\%) [45,46], while from India the oil was dominated by curcumenol (38.7\%) and $\beta$-pinene (27.5\%) [15] (Table 4). A C. aeruginosa oil sample from Thailand was dominated by curzerenone (41.6\%) followed by 1,8-cineole $(9.6 \%)$ and $\beta$-pinene $(7.7 \%)$ [38], whereas another sample had camphor $(29.4 \%)$, germacrone $(21.2 \%)$, isoborneol $(7.3 \%)$, germacrene B (5.2\%), and curzerene (4.8\%) [4]. 
Table 1. Chemical compositions (major components) of Curcuma longa rhizome volatile oils.

\begin{tabular}{|c|c|c|c|c|c|c|c|c|c|c|c|c|c|c|}
\hline Sample & 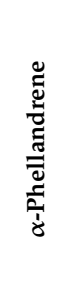 & 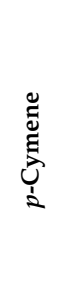 & 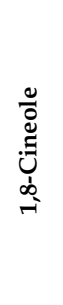 & 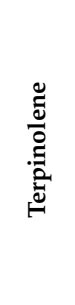 & 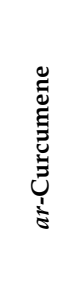 & 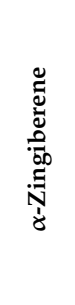 & 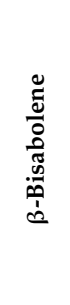 & 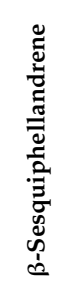 & 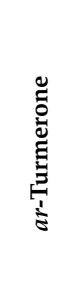 & 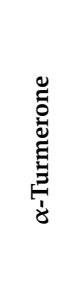 & 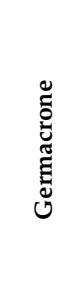 & 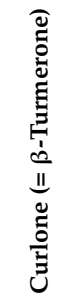 & 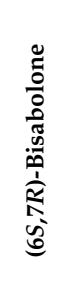 & 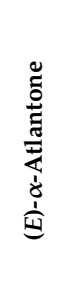 \\
\hline fRh-SD-India (APRC) & 2.41 & 2.44 & 3.22 & 0.53 & 0.89 & 0.34 & 0.29 & 0.98 & 36.02 & 16.95 & 0 & 19.07 & 0.81 & 0.38 \\
\hline Resin-SD-India (APRC) & 0.03 & 0.07 & 0.10 & 0 & 9.75 & 12.75 & 2.54 & 14.59 & 15.02 & 17.67 & 0 & 9.11 & 0.17 & 0.71 \\
\hline Rh-SD-Nepal (APRC) & 1.08 & 0.41 & 1.17 & 0.17 & 3.26 & 5.97 & 1.01 & 7.72 & 21.20 & 28.80 & 0 & 18.20 & 1.18 & 2.68 \\
\hline Rh-SD-Nepal (APRC) & 0.43 & 0.32 & 1.55 & 10.22 & 2.15 & 5.02 & 0.87 & 5.48 & 20.51 & 26.73 & 0 & 12.99 & 0.87 & 1.71 \\
\hline Rh-SD-Indonesia (APRC) & 2.94 & 0.67 & 0.45 & 0.57 & 3.14 & 2.77 & 0.75 & 3.38 & 14.54 & 15.41 & 0 & 10.03 & 0 & 0.90 \\
\hline Rh- $\mathrm{CO}_{2}$-India (APRC) & 0.41 & 0.45 & 0.35 & 0 & 3.87 & 3.35 & 0.82 & 4.69 & 33.82 & 20.62 & 0 & 14.42 & 1.29 & 3.26 \\
\hline $\mathrm{Rh}-\mathrm{CO}_{2}$-India (APRC) & 0.28 & 0.44 & 1.05 & 0 & 5.66 & 4.20 & 1.35 & 8.08 & 32.02 & 13.22 & 0 & 14.70 & 1.46 & 2.59 \\
\hline Rh- $-\mathrm{CO}_{2}$-India (APRC) & 0.61 & 0.45 & 1.89 & 0 & 4.41 & 6.29 & 1.49 & 9.15 & 25.23 & 19.96 & 0 & 14.63 & 1.16 & 2.02 \\
\hline Rh-SD-Indonesia (APRC) & 3.14 & 0.66 & 0.77 & 0.50 & 1.30 & 1.80 & 0.30 & 1.95 & 21.51 & 27.90 & 0 & 17.65 & 1.17 & 1.90 \\
\hline Rh-SD-Indonesia (APRC) & 3.10 & 1.19 & 1.15 & 0.76 & 11.07 & 3.23 & 2.16 & 8.44 & 17.45 & 13.17 & 0 & 8.81 & 0.62 & 0.72 \\
\hline Rh-HD-Jamaica (APRC) & 2.20 & 0.70 & 1.77 & 0.66 & 1.28 & 1.76 & 0.35 & 1.76 & 22.19 & 34.24 & 0 & 16.40 & 0.74 & 0.30 \\
\hline Rh-Unknown (APRC) & 0.45 & 0.26 & 1.14 & 0.30 & 2.46 & 4.94 & 0.79 & 4.71 & 25.32 & 25.30 & 0 & 17.49 & 0.88 & 1.04 \\
\hline Rh-SD-India (APRC) & 0.31 & 0.47 & 1.44 & 0 & 5.50 & 4.71 & 1.46 & 7.77 & 32.12 & 15.06 & 0 & 13.18 & 1.27 & 2.11 \\
\hline Rh-Unknown (APRC) & 1.25 & 1.40 & 4.04 & 0 & 6.59 & 6.12 & 1.57 & 9.51 & 29.00 & 13.38 & 0 & 11.97 & 1.07 & 1.50 \\
\hline Rh-SD-Indonesia (APRC) & 1.58 & 0.50 & 0.91 & 0.32 & 2.05 & 2.41 & 0.43 & 2.39 & 21.95 & 31.05 & 0 & 18.86 & 0.71 & 1.09 \\
\hline Rh-SD-Nepal (APRC) & 3.79 & 2.52 & 2.24 & 1.52 & 4.09 & 6.08 & 1.15 & 3.20 & 21.84 & 20.21 & 0 & 9.72 & 0.38 & 1.63 \\
\hline Rh-SD-Nepal (APRC) & 0.05 & 0 & 0.23 & 1.58 & 0.99 & 8.81 & 0.94 & 5.65 & 12.52 & 44.51 & 0 & 14.44 & 1.06 & 0.26 \\
\hline dRh-SD-India (APRC) & 3.36 & 2.31 & 0.79 & 0.33 & 2.62 & 1.42 & 0.44 & 2.03 & 36.64 & 23.73 & 0 & 15.74 & 0.66 & 0.19 \\
\hline fRh-SD-India (APRC) & 1.13 & 0.44 & 0.35 & 0.21 & 2.65 & 3.64 & 0.74 & 2.94 & 28.77 & 26.50 & 0 & 13.68 & 0.61 & 2.31 \\
\hline Rh-SD-Nepal (APRC) & 0 & 0.10 & 0.26 & 1.11 & 1.12 & 0.48 & 0.18 & 1.13 & 36.37 & 12.57 & 10.29 & 12.22 & 1.10 & 0.58 \\
\hline Rh-HD-Nepal (APRC) & 0.03 & 0.09 & 0.38 & 1.54 & 0.87 & 0.54 & 0 & 1.06 & 35.07 & 20.50 & 11.11 & 14.18 & 0.95 & 0.26 \\
\hline Rh-Unknown (APRC) & 0.05 & 0.03 & 0.20 & 1.54 & 0.95 & 8.58 & 0.83 & 5.51 & 12.19 & 43.30 & 0 & 14.11 & 1.03 & 0.21 \\
\hline Rh-Unknown (APRC) & 6.73 & 0.79 & 1.49 & 0.41 & 1.70 & 3.30 & 0.43 & 2.72 & 18.20 & 37.75 & 0 & 37.75 & 0.40 & 0.64 \\
\hline Rh-SD-Nepal (APRC) & 0.16 & 0.09 & 0.30 & 4.38 & 1.69 & 4.28 & 0.68 & 4.73 & 23.18 & 28.93 & 0 & 14.76 & 1.13 & 2.38 \\
\hline $\mathrm{Rh}-\mathrm{CO}_{2}$-India (APRC) & 0.36 & 0.52 & 1.26 & 0 & 5.65 & 4.13 & 1.25 & 8.00 & 35.08 & 13.67 & 0 & 15.06 & 1.31 & 2.49 \\
\hline $\mathrm{Rh}-\mathrm{CO}_{2}$-India (APRC) & 0 & 0 & 0.3 & 0 & 3.13 & 2.78 & 0.76 & 4.43 & 34.20 & 21.49 & 0 & 16.34 & 0 & 3.77 \\
\hline Rh-SD-Nepal (APRC) & 0.16 & 0.16 & 0.18 & 6.04 & 0 & 2.56 & 0.27 & 2.15 & 27.36 & 32.11 & 0.92 & 16.72 & 1.27 & 0.63 \\
\hline dRh-SD-India (APRC) & 1.13 & 0.98 & 1.20 & 8.91 & 6.14 & 5.98 & 1.50 & 3.17 & 32.16 & 9.39 & 0 & 3.96 & 0 & 0 \\
\hline Rh-HD-Nepal (APRC) & 0 & 0.07 & 0.39 & 1.54 & 0.90 & 0.66 & 0.14 & 1.07 & 34.42 & 20.25 & 11.10 & 13.90 & 0.98 & 0.29 \\
\hline Rh-HD-Nepal (APRC) & 0.01 & 0.01 & 0.13 & 0.76 & 1.71 & 4.53 & 0.70 & 4.04 & 23.68 & 35.42 & 0 & 14.43 & 1.04 & 0.28 \\
\hline Rh-HD-Nepal (APRC) & 0.41 & 0.30 & 1.51 & 10.01 & 2.10 & 4.90 & 0.82 & 5.35 & 20.12 & 26.20 & 0 & 12.72 & 0.83 & 1.67 \\
\hline Rh-HD-Nepal (APRC) & 0.03 & 0.08 & 0.49 & 2.87 & 1.29 & 1.87 & 0.37 & 2.67 & 31.45 & 26.92 & 0 & 15.65 & 1.14 & 0.36 \\
\hline
\end{tabular}

$\mathrm{Rh}=$ rhizome; $\mathrm{dRh}=$ dried rhizome; $\mathrm{fRh}=$ fresh rhizome; $\mathrm{HD}=$ hydrodistillation; $\mathrm{SD}=$ steam distillation; $\mathrm{CO}_{2}=$ supercritical $\mathrm{CO}_{2}$ extracts; $\mathrm{APRC}=$ from the collection of the Aromatic Plant Research Center. 
Table 2. Chemical composition of Curcuma aromatica rhizome essential oils.

\begin{tabular}{|c|c|c|c|c|c|c|c|c|c|c|c|}
\hline Compound & $\begin{array}{c}\text { Car India } \\
\text { [15] }\end{array}$ & $\begin{array}{c}\text { Car India } \\
\text { [42] }\end{array}$ & $\begin{array}{c}\text { Car India } \\
\text { [42] }\end{array}$ & $\begin{array}{c}\text { Car Thailand } \\
{[38]}\end{array}$ & $\begin{array}{c}\text { Car Japan } \\
\text { [37] }\end{array}$ & $\begin{array}{c}\text { Car Japan } \\
\text { [37] }\end{array}$ & $\begin{array}{c}\text { Car India } \\
\text { [37] }\end{array}$ & $\begin{array}{c}\text { Car India } \\
\text { [39] }\end{array}$ & $\begin{array}{c}\text { Car India } \\
\text { [39] }\end{array}$ & $\begin{array}{c}\text { Car India } \\
\text { [40] }\end{array}$ & $\begin{array}{c}\text { Car India } \\
\text { [41] }\end{array}$ \\
\hline$\alpha$-Pinene & 1.5 & 5.9 & 5.7 & 0.5 & 0.4 & 0.9 & 0.2 & 0.4 & 0.3 & 0.3 & 0.8 \\
\hline Camphene & 10.2 & 0.9 & 1.1 & 2.0 & 0 & 0 & 0.3 & 0.9 & 0.8 & 0.7 & 7.4 \\
\hline Myrcene & 1.2 & 0 & 0 & 0.4 & 0.1 & 0.3 & 0.1 & 0.2 & 0.2 & 0.2 & 1.0 \\
\hline 1,8-Cineole & 10.1 & 13.7 & 15.9 & 0.3 & 7.5 & 25.3 & 1.0 & 0.1 & 0.1 & 5.5 & 9.3 \\
\hline Terpinolene & 0 & 5.2 & 3.9 & 0 & 0 & 0 & 0 & $\operatorname{tr}$ & $\operatorname{tr}$ & 0 & 0.1 \\
\hline Linalool & 2.1 & 0 & 0 & 0.6 & 2.2 & 2.8 & 0.1 & 0 & 0 & 0.2 & 1.2 \\
\hline Camphor & 18.8 & 48.3 & 45.7 & 26.9 & 0 & 0 & 3.9 & 3.9 & 3.3 & 32.3 & 25.6 \\
\hline Isoborneol & 1.8 & 12.2 & 10.1 & 2.3 & 0 & 0 & 0.3 & 0 & 0 & 3.4 & 8.2 \\
\hline Borneol & 8.2 & 5.0 & 4.9 & 1.7 & 0 & 0 & 0.3 & 1.8 & 1.1 & 1.1 & 2.5 \\
\hline$\alpha$-Terpineol & 0 & 0 & 0 & 0 & 0.4 & 1.3 & 1.4 & 0 & $\operatorname{tr}$ & 0.6 & 1.0 \\
\hline$\beta$-Elemene & 7.5 & 0 & 0 & 0.1 & 4.0 & 2.5 & 1.0 & 0.2 & 0.2 & 1.4 & 1.4 \\
\hline$\beta$-Caryophyllene & 2.0 & 0 & 0 & 0 & 1.9 & 1.7 & 0.3 & 0 & 0 & 0.3 & 0.3 \\
\hline$\alpha$-Humulene & 0 & 0 & 0 & 1.9 & 2.1 & 1.0 & 0 & 0 & 0 & $\operatorname{tr}$ & $\operatorname{tr}$ \\
\hline$\beta$-Farnesene & 0 & 0 & 0 & 0 & 0 & 0 & 2.6 & 0 & 0 & $\operatorname{tr}$ & 0 \\
\hline ar-Curcumene & 0 & 0 & 0 & 23.2 & 0 & 0 & 22.1 & 23.6 & 24.1 & 3.1 & 0 \\
\hline Germacrene D & 1.8 & 0 & 0 & 0 & 1.1 & 0.7 & 0.2 & $\operatorname{tr}$ & 0.3 & 0 & 0.9 \\
\hline Curzerene & 0 & 0.3 & 0.4 & 1.4 & 0 & 0 & 3.2 & 4.6 & 6.0 & 0.2 & 2.7 \\
\hline$\beta$-Curcumene & 0 & 0 & 0 & 3.9 & 0 & 0 & 29.9 & 28.4 & 31.4 & 0 & 0 \\
\hline Germacrene B & 2.8 & 0.2 & 0.4 & 0.9 & 0 & 0 & 0 & 0 & 0 & 0.3 & 0.4 \\
\hline Caryophyllene oxide & 0 & 0 & 0 & 0 & 1.4 & 2.0 & 0 & 0 & 0 & $\operatorname{tr}$ & $\operatorname{tr}$ \\
\hline Curzerenone & 0 & 0 & 0 & 3.8 & 0 & 0 & 3.6 & 7.3 & 5.5 & 11.0 & 10.9 \\
\hline Germacrone & 0 & 0.3 & 0.3 & 0.3 & 9.6 & 4.6 & 4.9 & 3.6 & 6.1 & 0.5 & 10.6 \\
\hline Xanthorrhizol & 4.8 & 0 & 0 & 18.7 & 0 & 0 & 16.2 & 8.0 & 5.3 & 0 & 0 \\
\hline Curdione & 8 & 4.8 & 6.8 & 0 & 44.0 & 32.2 & 0 & 0 & 0 & 0 & 0 \\
\hline
\end{tabular}


Table 3. Chemical composition of Curcuma zedoaria rhizome essential oils.

\begin{tabular}{|c|c|c|c|c|c|}
\hline Compound & $\begin{array}{c}\text { Cz Nepal-1 } \\
\text { (APRC) }\end{array}$ & $\begin{array}{c}\text { Cz Nepal-2 } \\
\text { (APRC) }\end{array}$ & $\begin{array}{l}\text { Cz India } \\
\text { (APRC) }\end{array}$ & Cz India [15] & Cz India [43] \\
\hline 1,8-Cineole & 8.77 & 38.39 & 7.00 & 0 & 1.9 \\
\hline Camphor & 8.79 & 0 & 8.26 & 3.3 & 10.3 \\
\hline Borneol/Isoborneol & 1.81 & 0.07 & 3.17 & 0.2 & 2.7 \\
\hline$\alpha$-Terpineol & 1.49 & 1.17 & 0.47 & 1.7 & 0.3 \\
\hline$\alpha$-Terpinyl acetate & 2.29 & 0 & 0 & 0 & 0 \\
\hline$\alpha$-Copaene & 17.35 & 0.42 & 0 & 0 & 0 \\
\hline$\beta$-Elemene & 2.89 & 0.21 & 5.54 & 0.3 & $\operatorname{tr}$ \\
\hline$\beta$-Caryophyllene & 8.28 & 1.37 & 1.46 & 0 & 0.4 \\
\hline$\gamma$-Elemene & 0.29 & 0 & 0.84 & 2.5 & 0.1 \\
\hline ar-Curcumene & 0 & 0.51 & 0 & 12.1 & 0 \\
\hline Zingiberene & 0 & 0 & 0 & 12.0 & 0 \\
\hline Curzerene & 2.36 & 0 & 5.93 & 8.0 & 0 \\
\hline$\alpha$-Farnesene & 0 & 0 & 0 & 2.3 & 0 \\
\hline$\gamma$-Cadinene & 0 & 2.20 & 0 & 0 & 0 \\
\hline$\delta$-Cadinene & 3.83 & 3.85 & 0.25 & 0 & 0 \\
\hline Germacrene B & 0.38 & 0 & 1.08 & 6.0 & 0.6 \\
\hline$\beta$-Sesquiphellandrene & 0 & 0 & 0 & 9.8 & 0 \\
\hline Elemol & 0 & 6.84 & 0 & 0 & 0 \\
\hline Curzerenone/epi-Curzerenone & 20.89 & 0 & 29.41 & 19.0 & 31.6 \\
\hline Germacrone & 2.59 & 0 & 6.65 & 0 & 10.8 \\
\hline Curlone (= $\beta$-Turmerone) & 0 & 0 & 0 & 4.0 & 0 \\
\hline Curdione & 0.10 & 0 & 1.23 & 0 & 1.3 \\
\hline Curcumenol & 0 & 0 & 1.57 & 0 & 2.2 \\
\hline
\end{tabular}

Table 4. Chemical composition of Curcuma aeruginosa rhizome essential oils reported in the literature.

\begin{tabular}{|c|c|c|c|c|c|}
\hline Compound & Cae Thailand [4] & Cae India [15] & Cae Thailand [38] & Cae Malaysia [46] & Cae Malaysia [45] \\
\hline Camphene & 1.2 & 0.18 & 0.3 & 1.6 & 0.2 \\
\hline$\beta$-Pinene & 0.4 & 27.5 & 7.7 & 1.6 & 0.4 \\
\hline 1,8-Cineole & 2.7 & 0.42 & 9.6 & 25.2 & 11.2 \\
\hline Camphor & 29.4 & 0 & 0 & 6.8 & 10.5 \\
\hline Isoborneol & 7.3 & 0 & 0.6 & 1.5 & 3.2 \\
\hline Borneol & 2.9 & 0 & 0.5 & 0.5 & 1.3 \\
\hline$\beta$-Elemene & 1.4 & 0 & 0.2 & 1.7 & 2.2 \\
\hline$\beta$-Farnesene & 0 & 1.5 & 0 & 0.5 & 1.0 \\
\hline Zingiberene & 0 & 1.2 & 0 & 0.1 & 0 \\
\hline Curzerene & 4.8 & 0 & 1.1 & 0 & 0 \\
\hline Germacrene B & 5.2 & 0 & 0.5 & 0 & 0 \\
\hline Curzerenone & 0 & 0 & 41.6 & 30.4 & 24.6 \\
\hline$\beta$-Eudesmol & 0 & 3.6 & 0 & 0 & 0 \\
\hline Germacrone & 21.2 & 0 & 1.0 & 2.8 & 2.7 \\
\hline Curcumenol & 0 & 38.7 & 0 & 0 & 5.6 \\
\hline
\end{tabular}

A hierarchical cluster analysis was carried out based on the $C$. longa essential oil compositions. For comparison, we included $C$. longa rhizome oils that were reported in the literature in this analysis, including 23 steam- or hydrodistilled essential oils and two supercritical $\mathrm{CO}_{2}$ extracts (Table 5). Although $C$. longa rhizome oils were all rich in ar-turmerone, $\alpha$-turmerone, and $\beta$-turmerone, the cluster analysis revealed four clearly defined clusters based on the relative concentrations of these major components (Figure 1). The cluster centroids of the major components of $C$. longa rhizome oils are summarized in Table 6, illustrating the chemical differences in the four clusters. Cluster 2 was the largest, representing 21 samples dominated by the turmerones (particularly ar-turmerone). Cluster 1 represents samples with relatively large concentrations of components other than turmerones; therefore, lower concentrations of turmerones. The third cluster was also a large cluster, representing 15 samples dominated by the turmerones (predominantly $\alpha$-turmerone). The fourth cluster had very large concentrations of ar-turmerone. 
Table 5. Chemical composition of Curcuma longa rhizome volatile oils from the published literature.

\begin{tabular}{|c|c|c|c|c|c|c|c|c|c|c|c|c|c|c|}
\hline Sample & 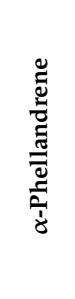 & 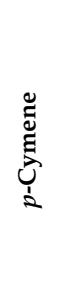 & 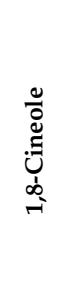 & 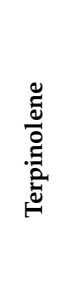 & 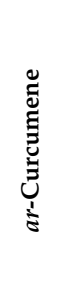 & 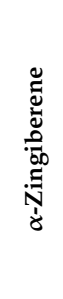 & 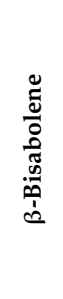 & 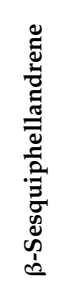 & 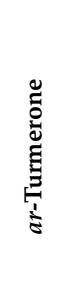 & 节 & 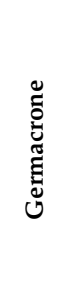 & 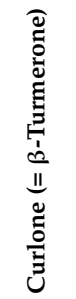 & 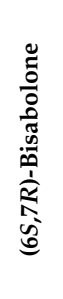 & 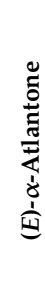 \\
\hline fRh-HD-Nigeria [47] & 15.5 & 2.1 & 10.3 & 3.2 & 0.7 & 2.0 & 0 & 1.8 & 10.0 & 35.9 & 0 & 12.9 & 0 & 0 \\
\hline dRh-HD-China [48] & 0 & 0.5 & 0.5 & 0.3 & 6.1 & 20.1 & 5.1 & 15.5 & 27.5 & 0.1 & 0.3 & 1.7 & 0 & 0 \\
\hline fRh-HD-India [15] & 3.1 & 0.3 & 0.7 & 0.1 & 3.5 & 4.0 & 0 & 0.8 & 49.8 & 9.1 & 0 & 7.9 & 0 & 0 \\
\hline dRh-HD-Iran [49] & 2.2 & 0.4 & 0.4 & 1.5 & 0.8 & 1.5 & 0.4 & 1.3 & 68.9 & 20.9 & 0 & 0 & 0 & 0 \\
\hline fRh-HD-India [50] & 0.1 & 0.3 & 0.4 & 2.7 & 1.6 & 2.5 & 0.8 & 2.9 & 24.4 & 20.5 & 1.0 & 11.1 & 1.7 & 0.9 \\
\hline dRh-HD-India [50] & 0 & 0.1 & 0.1 & $\operatorname{tr}$ & 6.6 & 0.8 & 4.1 & 4.2 & 21.4 & 0.6 & 2.6 & 4.3 & 0.8 & 2.6 \\
\hline fRh-HD-India [51] & 2.0 & 0.6 & 0.8 & 0.2 & 1.9 & 2.6 & 0.4 & 2.4 & 21.0 & 33.5 & 0 & 18.9 & 0 & 0 \\
\hline dRh-HD-India [51] & $\operatorname{tr}$ & 0 & $\operatorname{tr}$ & 0 & 1.2 & 2.2 & 1.5 & 2.8 & 30.3 & 26.5 & 0 & 19.1 & 0 & 0 \\
\hline fRh-HD-India [52] & 8.0 & 4.3 & 11.2 & 0.7 & 4.4 & 5.6 & 2.8 & 7.1 & 7.3 & 11.1 & 0.1 & 5.0 & 0.1 & 0.2 \\
\hline fRh-HD-India [53] & 9.4 & 1.2 & 1.9 & 1.2 & 0.5 & 2.3 & 0 & 1.8 & 5.4 & 44.1 & 0.4 & 18.5 & 0 & 1.1 \\
\hline fRh-HD-India [54] & 0.1 & 0.1 & 2.6 & 0.1 & 0.2 & 1.3 & 0.2 & 0 & 31.7 & 12.9 & 0.9 & 12.0 & 0.2 & 1.5 \\
\hline dRh-HD-India [55] & 2.2 & 1.0 & 0 & 0 & 4.8 & 0 & 0 & 0 & 53.1 & 6.2 & 0 & 6.4 & 0 & 0 \\
\hline dRh-HD-Thailand [49] & 2.2 & 0.4 & 0.4 & 1.5 & 0.8 & 1.5 & 0.4 & 1.3 & 68.9 & 20.9 & 0 & 0 & 0 & 0 \\
\hline fRh-HD-Pakistan [56] & 0.4 & 0 & 1.6 & 0 & 0 & 0 & 0 & 0 & 25.3 & 18.4 & 0 & 12.5 & 0 & 0 \\
\hline fRh-HD-Bangladesh [57] & 0.5 & 0.2 & 0 & 0 & 3.3 & 4.4 & 0.2 & 5.6 & 27.8 & 17.2 & 0 & 13.8 & 0 & 0 \\
\hline fRh-SD-Bhutan [58] & 1.7 & 0.5 & 7.6 & 0.7 & 1.4 & 4.2 & 0.7 & 3.6 & 16.7 & 30.1 & 0 & 14.7 & 1.0 & 1.2 \\
\hline fRh-HD-Brazil [59] & 6.5 & 0.9 & 3.2 & 1.4 & 1.0 & 1.9 & 0.3 & 1.4 & 12.9 & 42.6 & 0.5 & 16.0 & 0.3 & 0.5 \\
\hline dRh-HD-Brazil [60] & 1.7 & 0.8 & 0.7 & 0 & 2.6 & 1.0 & 0 & 2.4 & 33.2 & 23.5 & 0 & 22.7 & 3.1 & 1.4 \\
\hline dRh-HD-S. Tomé e Principe [35] & 15.5 & 2.5 & 10.2 & 3.1 & 0.8 & 1.1 & 0 & 1.0 & 12.8 & 23.9 & 0 & 11.5 & 0 & 0.6 \\
\hline dRh-HD-S. Tomé e Principe [35] & 30.4 & 5.5 & 23.0 & 4.5 & 1.1 & 2.4 & 0 & 2.0 & 4.0 & 12.2 & 0 & 4.3 & 0 & 0 \\
\hline dRh-HD-Brazil [33] & 2.7 & 0 & 1.4 & 0 & 1.0 & 2.4 & $\operatorname{tr}$ & 1.9 & 18.0 & 44.0 & 0 & 18.3 & 0.6 & 0.6 \\
\hline fRh-SD-Reunion [61] & 1 & 0.6 & 2 & 15.8 & 4.5 & 11.8 & 1.9 & 8.8 & 7.7 & 21.4 & 0 & 7.1 & 0 & 0 \\
\hline fRh-HD-India [62] & 5.3 & 0 & 2.6 & 0 & 3.5 & 0 & 0.6 & 1.7 & 49.1 & 0 & 0 & 16.8 & 0 & 0 \\
\hline dRh-HD-India [63] & 1.8 & 1.3 & 1.3 & 0 & 1.4 & 1.7 & 0 & 1.7 & 34.0 & 34.0 & 0 & 15.0 & 0 & 0 \\
\hline dRh-HD-India [63] & 1.4 & 0.9 & 1.3 & 0 & 1.5 & 1.9 & 0 & 1.9 & 35.0 & 35.0 & 0 & 12.0 & 0 & 0 \\
\hline Rh- $-\mathrm{CO}_{2}$-Brazil [64] & 4.1 & 1.5 & 4.0 & 1.3 & 3.6 & 6.4 & 1.7 & 7.7 & 15.5 & 20.3 & 0 & 15.6 & 0.3 & 0.6 \\
\hline dRh- $-\mathrm{CO}_{2}$-China [65] & 0 & 0 & 0 & 2.2 & 1.9 & 16.9 & 1.5 & 10.0 & 11.0 & 40.8 & 0 & 14.1 & 0 & 0 \\
\hline
\end{tabular}

$\mathrm{Rh}=$ rhizome; $\mathrm{dRh}=$ dried rhizome; $\mathrm{fRh}=$ fresh rhizome; $\mathrm{HD}=$ hydrodistillation; $\mathrm{SD}=$ steam distillation; $\mathrm{CO}_{2}=$ supercritical $\mathrm{CO}_{2}$ extracts 
Curcuma longa Dendrogram

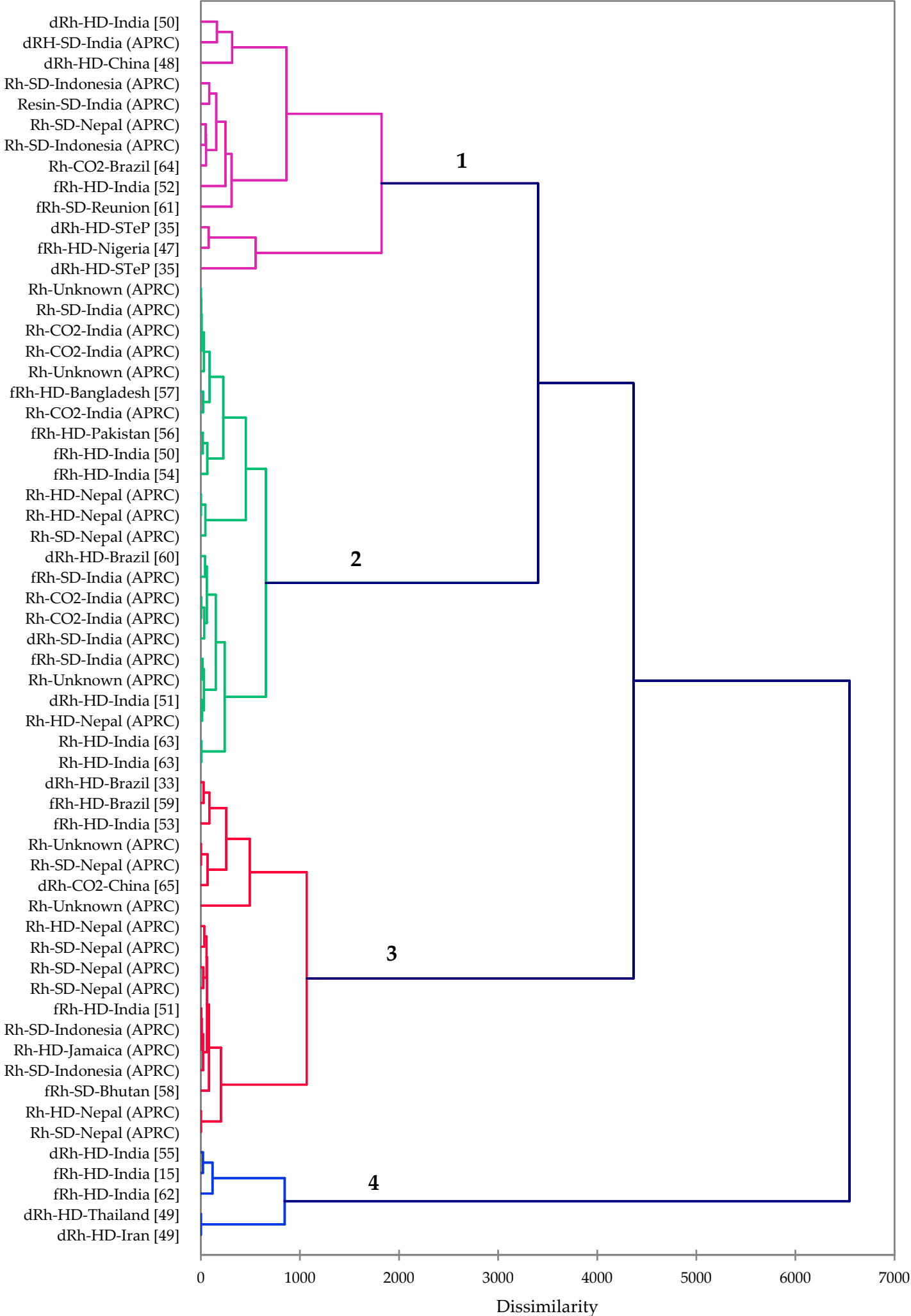

Figure 1. Dendrogram obtained from the agglomerative hierarchical cluster analysis of 60 Curcuma longa volatile oil samples. 
Table 6. Concentration (\%) of centroids used in the cluster analysis of Curcuma longa rhizome oils.

\begin{tabular}{lcccc}
\hline Compound & Cluster $\mathbf{1}$ & Cluster $\mathbf{2}$ & Cluster 3 & Cluster 4 \\
\hline$\alpha$-Phellandrene & 6.58 & 0.71 & 2.13 & 2.99 \\
1,8-Cineole & 5.11 & 1.11 & 1.39 & 0.82 \\
ar-Curcumene & 4.77 & 2.72 & 1.49 & 2.68 \\
$\alpha$-Zingiberene & 6.23 & 2.72 & 4.68 & 1.4 \\
$\beta$-Sesquiphellandrene & 6.22 & 3.89 & 3.92 & 1.02 \\
ar-Turmerone & 15.94 & 31.68 & 18.31 & 57.96 \\
$\alpha$-Turmerone & 15.49 & 20.56 & 35.11 & 11.41 \\
Curlone & 8.01 & 14.75 & 17.20 & 6.22 \\
\hline
\end{tabular}

Hierarchical cluster analysis of $C$. aromatica essential oils clearly identified three clusters based on dissimilarity (Figure 2). Cluster 1 had a relatively high camphor concentration, represented by the $C$. aromatica EO sample from Thailand [38]; cluster 2 was dominated by curdione followed by 1,8-cineole, represented by two samples from Japan [37]; and cluster 3 represents samples with large concentrations of ar-curcumene and $\beta$-curcumene [15,37,39-42]. Table 7 summarizes the cluster centroids of the major components of $C$. aromatica rhizome oils.

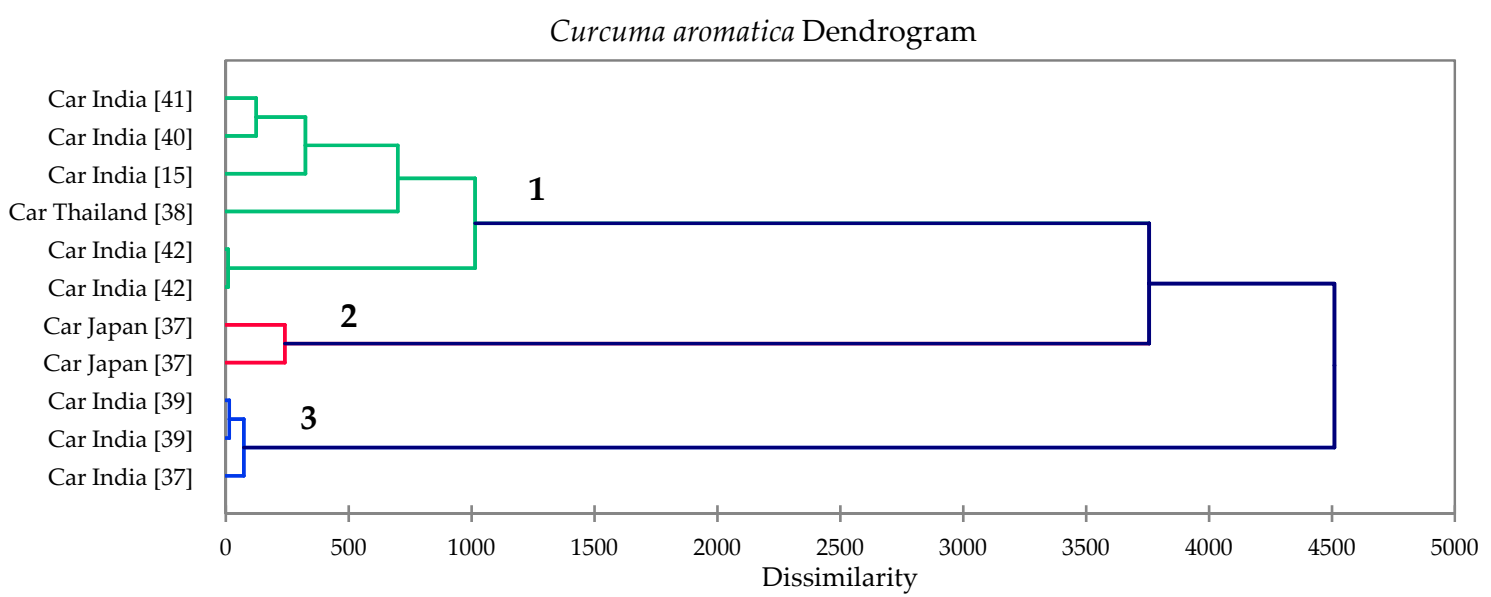

Figure 2. Dendrogram obtained from the agglomerative hierarchical cluster analysis of eight Curcuma aromatica essential oil samples.

Table 7. Concentration (\%) of centroids used in the cluster analysis of Curcuma aromatica rhizome oils.

\begin{tabular}{lccc}
\hline Compound & Cluster $\mathbf{1}$ & Cluster 2 & Cluster 3 \\
\hline Camphor & 28.28 & 0 & 3.69 \\
ar-Curcumene & 8.76 & 0 & 23.25 \\
Curdione & 0 & 38.08 & 0 \\
3-Curcumene & 1.30 & 0 & 29.93 \\
1,8-Cineole & 5.02 & 16.41 & 0.38 \\
Xanthorrhizol & 6.23 & 0 & 9.83 \\
Curzerenone & 8.57 & 0 & 5.43 \\
Germacrone & 3.80 & 7.09 & 4.85 \\
\hline
\end{tabular}

For C. zedoaria essential oils, the cluster analysis showed two clusters based on dissimilarity (Figure 3): (1) a cluster dominated by curzerenone/epi-curzerenone followed by camphor, germacrone, 1,8-cineole, and $\alpha$-copaene; and (2) a cluster represented by a single sample with very large concentrations of 1,8-cineole. The cluster centroids of the main constituents of $C$. zedoaria rhizome oils are summarized in Table 8. 
Curcuma zedoaria Dendrogram

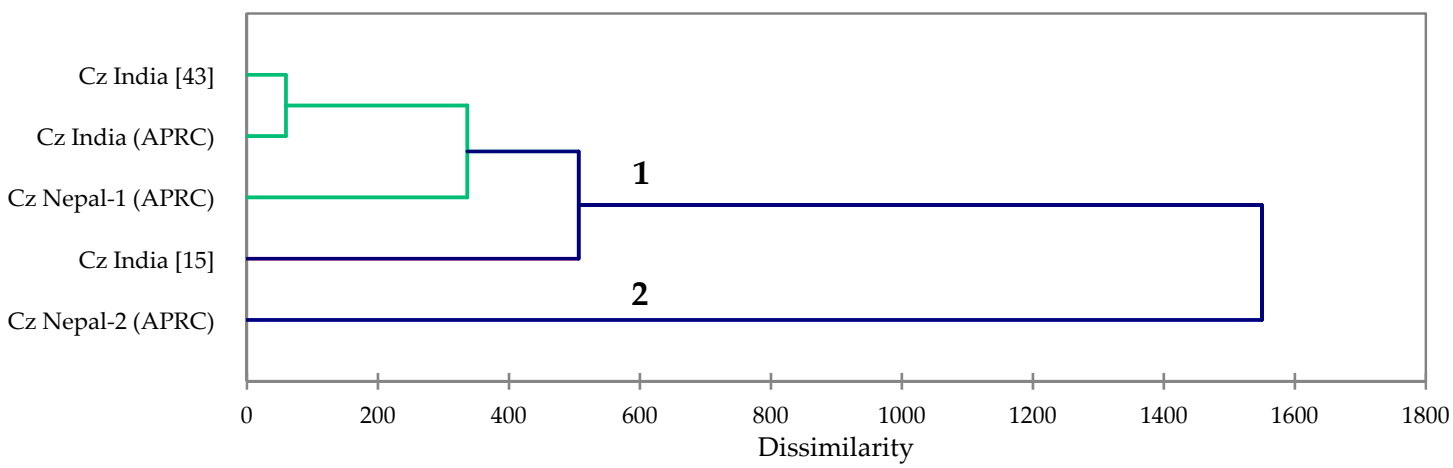

Figure 3. Dendrogram obtained from the agglomerative hierarchical cluster analysis of five Curcuma zedoaria essential oil samples.

Table 8. Concentration (\%) of centroids used in the cluster analysis of Curcuma aromatica rhizome oils.

\begin{tabular}{lcc}
\hline & Cluster 1 & Cluster 2 \\
\hline Curzerenone/epi-Curzerenone & 27.3 & 0 \\
1,8-Cineole & 4.42 & 38.39 \\
Camphor & 7.66 & 0 \\
Germacrone & 5.01 & 0 \\
$\alpha$-Copaene & 4.43 & 0.42 \\
Curzerene & 4.07 & 0 \\
ar-Curcumene & 3.03 & 0.51 \\
Zingiberene & 3.00 & 0 \\
$\beta$-Sesquiphellandrene & 2.45 & 0 \\
\hline
\end{tabular}

Curcuma aeruginosa essential oils showed three classes in hierarchical cluster analysis based on dissimilarity (Figure 4): (1) a camphor/germacrone rich cluster with large concentrations of isoborneol, curzerene, and germacrone B; (2) a curcumenol/ $\beta$-pinene rich cluster; and (3) a curzerenone/1,8-cineole cluster. Table 9 summarizes the concentrations of cluster centroids of the major components of $C$. aeruginosa rhizome oils. Although there are only five essential oil samples of C. zedoaria and C. aeruginosa, which is too few to give a comprehensive chemotaxonomic representation of these species, this analysis does provide initial insights into the potential chemotypes.

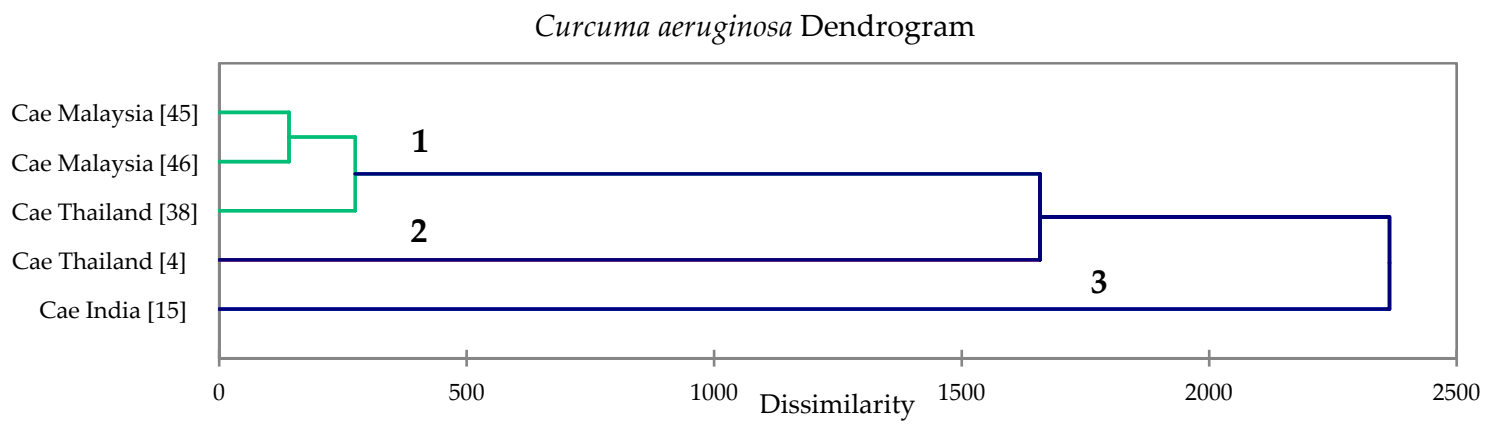

Figure 4. Dendrogram obtained from the agglomerative hierarchical cluster analysis of five Curcuma aeruginosa essential oil compositions. 
Table 9. Concentration (\%) of centroids used in the cluster analysis of Curcuma aeruginosa rhizome oils.

\begin{tabular}{lccc}
\hline & Cluster $\mathbf{1}$ & Cluster 2 & Cluster 3 \\
\hline Curzerenone & 0 & 0 & 32.21 \\
1,8-Cineole & 2.68 & 0.42 & 15.35 \\
Camphor & 29.39 & 0 & 5.77 \\
Curcumenol & 0 & 38.70 & 1.87 \\
$\beta$-Pinene & 0.35 & 27.50 & 3.24 \\
Germacrone & 21.21 & 0 & 2.16 \\
Isoborneol & 7.27 & 0 & 1.76 \\
Curzerene & 4.84 & 0 & 0.36 \\
Germacrene B & 5.20 & 0 & 0.17 \\
\hline
\end{tabular}

\section{Conclusions}

The rhizome essential oils of Curcuma longa, C. aromatica, C. zedoaria, and C. aeruginosa from the APRC collection, compared to the published literature, were analyzed by GC-MS. $\alpha$-Turmerone, curlone, ar-turmerone, $\beta$-sesquiphellandrene, $\alpha$-zingiberene, germacrone, terpinolene, ar-curcumene, and $\alpha$-phellandrene were the major components of C. longa. C. zedoaria rhizome oil contained 1,8-cineole, curzerenone/epi-curzerenone, $\alpha$-copaene, camphor, $\beta$-caryophyllene, elemol, germacrone, curzerene, and $\beta$-elemene. The cluster analysis revealed four clearly defined clusters for $C$. longa, three clusters for $C$. aromatica and C. aeruginosa, and two types for C. zedoaria.

In the case of $C$. longa, there are no apparent correlations based on extraction method (steam distillation, hydrodistillation, or supercritical $\mathrm{CO}_{2}$ extraction) or country or region of origin. Furthermore, the differences between the clusters are not that great, and therefore, the clusters do not likely represent distinct chemotypes but rather just reflect the chemical variation within each species. The data do provide a baseline for comparison of $C$. longa rhizome oils, however. These are important points when considering sources of either essential oils or rhizomes. There are still too few data to draw conclusions about the possible chemotypes of $C$. aromatica, C. aeruginosa, or C. zedoaria; more data are required.

Author Contributions: Conceptualization: N.S.D.; software: P.S.; validation: W.N.S.; formal analysis: W.N.S.; investigation: N.S.D., P.S., and W.N.S.; writing—original draft preparation: N.S.D.; writing—review and editing: N.S.D., P.S. and W.N.S.

Acknowledgments: This work was carried out as part of the activities of the Aromatic Plant Research Center (APRC, https:/ / aromaticplant.org/). We are grateful to Loren Bangerter for help with distillation of C. longa samples and to Prasun Satyal for supplying the Curcuma samples from Nepal.

Conflicts of Interest: The authors declare no conflict of interest.

\section{References}

1. Ravindran, P.N.; Babu, K.N.; Shiva, K.N. Botany and crop improvement of tumeric. In Turmeric: The Genus Curcuma; Ravindran, P.N., Babu, K.N., Sivaraman, K., Eds.; CRC Press: Boca Raton, FL, USA, 2007; pp. 15-70.

2. Chuakul, W.; Boonpleng, A. Ethnomedical uses of Thai Zingiberaceous plant (1). Thai J. Phytopharm. 2003, 10, 33-39.

3. Tushar; Basaka, S.; Sarma, G.C.; Rangan, L. Ethnomedical uses of Zingiberaceous plants of northeast India. J. Ethnopharmacol. 2010, 132, 286-296. [CrossRef] [PubMed]

4. Akarchariya, N.; Sirilun, S.; Julsrigival, J.; Chansakaowa, S. Chemical profiling and antimicrobial activity of essential oil from Curcuma aeruginosa Roxb., Curcuma glans K. Larsen \& J. Mood and Curcuma cf. xanthorrhiza Roxb. collected in Thailand. Asian Pac. J. Trop. Biomed. 2017, 7, 881-885.

5. Dosoky, N.S.; Setzer, W.N. Chemical composition and biological activities of essential oils of Curcuma species. Nutrients 2018, 10, 1196. [CrossRef] [PubMed]

6. Leong-Skornikova, J.; Newman, M. Gingers of Cambodia, Laos \& Vietnam; Oxford Graphic Printers Pte Ltd.: Singapore, 2015. 
7. Sikha, A.; Harini, A.; Prakash, H. Pharmacological activities of wild turmeric (Curcuma aromatica Salisb): A review. J. Pharmacogn. Phytochem. 2015, 3, 1-4.

8. Afzal, A.; Oriqat, G.; Khan, M.A.; Jose, J.; Afzal, M. Chemistry and biochemistry of terpenoids from Curcuma and related species. J. Biol. Act. Prod. Nat. 2013, 3, 1-55.

9. Krup, V.; Prakash, H.L.; Harini, A. Pharmacological activities of turmeric (Curcuma longa Linn): A review. J. Tradit. Med. Clin. Naturop. 2013, 2, 1206-2167. [CrossRef]

10. Herath, H.M.I.C.; Wiyasiriwardene, T.D.C.M.K.; Premakumara, G.A.S. Comparative GC-MS analysis of all Curcuma species grown in Sri Lanka by multivariate test. Ruhunu J. Sci. 2017, 8, 1-9. [CrossRef]

11. Chen, I.N.; Chang, C.C.; Ng, C.C.; Wang, C.Y.; Shyu, Y.T.; Chang, T.L. Antioxidant and antimicrobial activity of Zingiberaceae plants in Taiwan. Plants Food Hum. Nutr. 2008, 63, 15-20. [CrossRef]

12. Reanmongkol, W.; Subhadhirasakul, S.; Khaisombat, N.; Fuengnawakit, P.; Jantasila, S.; Khamjun, A. Investigation the antinociceptive, antipyretic and anti-inflammatory activities of Curcuma aeruginosa Roxb. extracts in experimental animals. Songklanakarin J. Sci. Technol. 2006, 28, 999-1008.

13. Wilson, B.; Abraham, G.; Manju, V.S.; Mathew, M.; Vimala, B.; Sundaresan, S.; Nambisan, B. Antimicrobial activity of Curcuma zedoaria and Curcuma malabarica tubers. J. Ethnopharmacol. 2005, 99, 147-151. [CrossRef] [PubMed]

14. Mau, J.; Lai, E.Y.C.; Wang, N.P.; Chen, C.C.; Chang, C.H.; Chyau, C.C. Composition and antioxidant activity of the essential oil from Curcuma zedoaria. Food Chem. 2003, 82, 583-591. [CrossRef]

15. Angel, G.R.; Menon, N.; Vimala, B.; Nambisan, B. Essential oil composition of eight starchy Curcuma species. Ind. Crops Prod. 2014, 60, 233-238. [CrossRef]

16. Simoh, S.; Zainal, A. Chemical profiling of Curcuma aeruginosa Roxb. rhizome using different techniques of solvent extraction. Asian Pac. J. Trop. Biomed. 2015, 5, 412-417. [CrossRef]

17. Lakshmi, S.; Padmaja, G.; Remani, P. Antitumour effects of isocurcumenol isolated from Curcuma zedoaria rhizomes on human and murine cancer cells. Int. J. Med. Chem. 2011, 2011, 253962. [PubMed]

18. Jayaprakasha, G.K.; Jagan, L.; Rao, M.; Sakariah, K.K. Chemistry and biological activity of Curcuma longa. Trend Food Sci. Technol. 2005, 16, 533-548. [CrossRef]

19. Kunnumakkara, A.B.; Anand, P.; Aggarwal, B.B. Curcumin inhibits proliferation, invasion, angiogenesis and metastasis of different cancers through interaction with multiple cell signaling proteins. Cancer Lett. 2008, 269, 199-225. [CrossRef] [PubMed]

20. Satoskar, R.R.; Shah, S.J.; Shenoy, S.G. Evaluation of antiinflammatory property of curcumin (diferuloyl methane) in patients with postoperative inflammation. Int. J. Clin. Pharma. Ther. Toxicol. 1986, 24, 651-654.

21. Masuda, T.; Kidaka, K.; Shinihara, A.; Mackawa, T.; Takeda, Y.; Yamaguchi, H. Chemical studies on antioxidant mechanism of curcuminoid: Analysis of radical reaction products from curcumin. J. Agric. Food Chem. 1999, 47, 71-77. [CrossRef]

22. Negi, P.S.; Jayaprakasha, G.K.; Jagan Mohan Rao, L.; Sakariah, K.K. Antibacterial activity of turmeric oil: A byproduct from curcumin manufacture. J. Agric. Food Chem. 1999, 47, 4297-4300. [CrossRef]

23. Jacob, J.N. Comparative studies in relation to the structure and biochemical properties of the active compounds in the volatile and nonvolatile fractions of turmeric (C. longa) and ginger (Z. officinale). In Studies in Natural Products Chemistry; Atta-ur-Rahman, Ed.; Elsevier: Amsterdam, The Netherlands, 2016; Volume 48, pp. 101-135.

24. Tilak, J.C.; Banerjee, H.M.; Mohan, T.P.A. Antioxidant availability of turmeric in relation to its medicinal and culinary uses. Phytother. Res. 2004, 18, 798-804. [CrossRef] [PubMed]

25. Park, S.Y.; Kim, D. Discovery of natural products from Curcuma longa that protect cells from beta-amyloid insult: A drug discovery effort against Alzheimer's disease. J. Nat. Prod. 2002, 65, 1227-1231. [CrossRef] [PubMed]

26. Weiss, E.A. Spice Crops; CAB International Publishing: Oxon, UK, 2002.

27. Gopalan, B.; Goto, M.; Kodama, A.; Hirose, T. Supercritical carbon dioxide extraction of turmeric (Curcuma longa). J. Agric. Food Chem. 2000, 48, 2189-2192. [CrossRef] [PubMed]

28. Sanghamitra, N.; Sujata, M.; Nagar, K. Differential effect of soil and environment on metabolic expression of turmeric (Curcuma longa cv. Roma). Indian J. Exp. Biol. 2015, 53, 406-411.

29. Srinivasan, V.; Thankamani, C.K.; Dinesh, R.; Kandiannan, K.; Zachariah, T.J.; Leela, N.K.; Hamza, S.; Shajina, O.; Ansha, O. Nutrient management systems in turmeric: Effects on soil quality, rhizome yield and quality. Ind. Crops Prod. 2016, 85, 241-250. [CrossRef] 
30. Dosoky, N.S.; Setzer, W.N. Biological activities and safety of Citrus spp. essential oils. Int. J. Mol. Sci. 2018, 19, 1966. [CrossRef] [PubMed]

31. Adams, R.P. Identification of Essential Oil Components by Gas Chromatography/Mass Spectrometry, 4th ed.; Allured Publishing: Carol Stream, IL, USA, 2007.

32. Satyal, P. Development of GC-MS Database of Essential Oil Components by the Analysis of Natural Essential Oils and Synthetic Compounds and Discovery of Biologically Active Novel Chemotypes in Essential Oils. Ph.D. Thesis, University of Alabama in Huntsville, Huntsville, AL, USA, 2015.

33. Braga, M.E.M.; Leal, P.F.; Carvalho, J.E.; Meireles, M.A.A. Comparison of yield, composition and antioxidant activity of turmeric (Curcuma longa L.) extracts obtained using various techniques. J. Agric. Food Chem. 2003, 51, 6604-6611. [CrossRef] [PubMed]

34. Usman, L.A.; Hamid, A.A.; George, O.C.; Ameen, O.M.; Muhammad, N.O.; Zubair, M.F.; Lawal, A. Chemical composition of rhizome essential oil of Curcuma longa L. growing in north central Nigeria. World J. Chem. 2009, 4, 178-181.

35. Martins, A.P.; Salgueiro, L.; Gonçalves, M.J.; da Cunha, A.P.; Vila, R.; Canigueral, S.; Mazzoni, V.; Tomi, F.; Casanova, J. Essential oil composition and antimicrobial activity of three Zingiberaceae from S. Tomé e Principe. Planta Med. 2001, 67, 580-584. [CrossRef] [PubMed]

36. Al-Reza, S.M.; Rahman, A.; Sattar, M.A.; Rahman, M.O.; Fida, H.M. Essential oil composition and antioxidant activities of Curcuma aromatica Salisb. Food Chem. Toxicol. 2010, 48, 1757-1760. [CrossRef]

37. Kojima, H.; Yanai, T.; Toyota, A. Essential oil constituents from Japanese and Indian Curcuma aromatica rhizomes. Planta Med. 1998, 64, 380-381. [CrossRef] [PubMed]

38. Jarikasem, S.; Thubthimthed, S.; Chawananoraseth, K.; Suntorntanasat, T. Essential oils from three Curcuma species collected in Thailand. Acta Hortic. 2005, 677, 37-41. [CrossRef]

39. Catalan, C.A.N.; Bardon, A.; Retamar, J.A.; Gros, E.G.; Verghese, J.; Joy, M.T. The essential oil of Curcuma aromatica Salisb. Flavor Fragr. J. 1989, 4, 25-30. [CrossRef]

40. Bordoloi, A.K.; Sperkova, J.; Leclercq, P.A. Essential oils of Curcuma aromatica Salisb. from northeast India. J. Essent. Oil Res. 1999, 11, 537-540. [CrossRef]

41. Choudhury, S.N.; Ghosh, A.C.; Saikia, M.; Choudhury, M.; Leclercq, P.A. Volatile constituents of the aerial and underground parts of Curcuma aromatica Salisb from India. J. Essent. Oil Res. 1996, 8, 633-638. [CrossRef]

42. Gopichand; Singh, R.D.; Meena, R.L.; Singh, M.K.; Kaul, V.K.; Lal, B.; Acharya, R.; Prasad, R. Effect of manure and plant spacing on crop growth, yield and oil-quality of Curcuma aromatica Salisb. in mid hill of western Himalaya. Ind. Crops Prod. 2006, 24, 105-112. [CrossRef]

43. Singh, P.; Singh, S.; Kapoor, I.P.S.; Singh, G.; Isidorov, V.; Szczepaniak, L. Chemical composition and antioxidant activities of essential oil and oleoresins from Curcuma zedoaria rhizomes, part-74. Food Biosci. 2013, 3, 42-48. [CrossRef]

44. Srivastava, S.; Chitranshi, N.; Srivastava, S.; Dan, M.; Rawat, A.K.S.; Pushpangadan, P. Pharmacognostic evaluation of Curcuma aeruginosa Roxb. Nat. Prod. Sci. 2006, 12, 162-165.

45. Sirat, M.H.; Jamil, S.; Hussain, J. Essential oil of Curcuma aeruginosa Roxb. from Malaysia. J. Essent. Oil Res. 1998, 10, 453-458. [CrossRef]

46. bin Jantan, I.; Ahmad, A.S.; Ali, N.A.M.; Ahmad, A.R.; Ibrahim, H. Chemical composition of the rhizome oils of four Curcuma species from Malaysia. J. Essent. Oil Res. 1999, 11, 719-723. [CrossRef]

47. Oyemitan, I.A.; Elusiyan, C.A.; Onifade, A.O.; Akanmu, M.A.; Oyedeji, A.O.; McDonald, A.G. Neuropharmacological profile and chemical analysis of fresh rhizome essential oil of Curcuma longa (turmeric) cultivated in southwest Nigeria. Toxicol. Rep. 2017, 4, 391-398. [CrossRef]

48. Zhang, L.; Yang, Z.; Chen, F.; Su, P.; Chen, D.; Pan, W.; Fang, Y.; Dong, C.; Zheng, X.; Du, Z. Composition and bioactivity assessment of essential oils of Curcuma longa L. collected in China. Ind. Crops Prod. 2017, 109, 60-73. [CrossRef]

49. Asghari, G.; Mostajeran, A.; Shebli, M. Curcuminoid and essential oil components of turmeric at different stages of growth cultivated in Iran. Res. Pharm. Sci. 2009, 4, 55-61.

50. Singh, G.; Kapoor, I.P.S.; Singh, P.; de Heluani, C.S.; de Lampasona, M.P.; Catalan, C.A.N. Comparative study of chemical composition and antioxidant activity of fresh and dry rhizomes of turmeric (Curcuma longa Linn.). Food Chem. Toxicol. 2010, 48, 1026-1031. [CrossRef] 
51. Kutti Gounder, D.; Lingamallu, J. Comparison of chemical composition and antioxidant potential of volatile oil from fresh, dried and cured turmeric (Curcuma longa) rhizomes. Ind. Crops Prod. 2012, 38, 124-131. [CrossRef]

52. Raina, V.K.; Srivastava, S.K.; Jain, N.; Ahmad, A.; Syamasundar, K.V.; Aggarwal, K.K. Essential oil composition of Curcuma longa L. cv. Roma from the plains of northern India. Flavour Fragr. J. 2002, 17,99-102. [CrossRef]

53. Raina, V.K.; Syamsundar, S.K.; Srivastava, K.V. Rhizome and leaf oil composition of Curcuma longa from the lower Himalayan region of northern India. J. Essent. Oil Res. 2005, 17, 556-559. [CrossRef]

54. Awasthi, P.K.; Dixit, S.C. Chemical composition of Curcuma longa leaves and rhizome oil from the plains of northern India. J. Young Pharm. 2009, 1, 312-316.

55. Naveen Kumar, K.; Venkataramana, M.; Allen, J.A.; Chandranayaka, S.; Murali, H.S.; Batra, H.V. Role of Curcuma longa L. essential oil in controlling the growth and zearalenone production of Fusarium graminearum. LWT Food Sci. Technol. 2016, 69, 522-528. [CrossRef]

56. Naz, S.; Ilyas, S.; Parveen, Z.; Javed, S. Chemical analysis of essential oils from turmeric (Curcuma longa) rhizome through GC-MS. Asian J. Chem. 2010, 22, 3153-3158.

57. Chowdhury, J.U.; Nandi, N.C.; Bhuiyan, M.N.I.; Mobarok, M.H. Essential oil constituents of the rhizomes of two types of Curcuma longa of Bangladesh. Bangladesh J. Sci. Ind. Res. 2008, 43, 259-266. [CrossRef]

58. Sharma, R.K.; Mishra, B.P.; Sharma, T.C.; Bordloi, A.K.; Pathak, M.G.; Leclercq, P.A. Essential oil of Curcuma longa L. from Bhutan. J. Essent. Oil Res. 1997, 9, 589-592. [CrossRef]

59. Avanço, G.B.; Ferreira, F.D.; Bomfimb, N.S.; de Souza Rodrigues dosSantos, P.A.; Peralta, R.M.; Brugnari, T.; Mallmann, C.A.; de Abreu Filho, B.A.; Mikcha, J.M.G.; Machinski, M., Jr. Curcuma longa L. essential oil composition, antioxidant effect, and effect on Fusarium verticillioides and fumonisin production. Food Control 2017, 73, 806-813.

60. Ferreira, F.D.; Kemmelmeier, C.; Arrotéia, C.C.; Da Costa, C.L.; Mallmann, C.A.; Janeiro, V.; Ferreira, F.M.D.; Mossini, S.A.G.; Silva, E.L.; Machinski, M. Inhibitory effect of the essential oil of Curcuma longa L. and curcumin on aflatoxin production by Aspergillus flavus Link. Food Chem. 2013, 136, 789-793. [CrossRef]

61. Chane-Ming, J.; Vera, R.; Chalchat, J.C.; Cabassu, P. Chemical composition of essential oils from rhizomes, leaves and flowers of Curcuma longa L. from Reunion Island. J. Essent. Oil Res. 2002, 14, 249-251. [CrossRef]

62. Singh, S.; Sankar, B.; Rajesh, S.; Sahoo, K.; Subudhi, E.; Nayak, S. Chemical composition of turmeric oil (Curcuma longa L. cv. Roma) and its antimicrobial activity against eye infecting pathogens. J. Essent. Oil Res. 2011, 23, 11-18. [CrossRef]

63. Chatterjee, S.; Variyar, P.S.; Gholap, A.S.; Bongirwar, D.R. Effect of $\gamma$-irradiation on the volatile oil constituents of turmeric (Curcuma longa). Food Res. Int. 2000, 33, 103-106. [CrossRef]

64. Chassagnez-mendez, A.L.; Machado, N.T.; Araujo, M.E.; Maia, J.G.; Meireles, A.A. Supercritical $\mathrm{CO}_{2}$ extraction of curcumins and essential oil from the rhizomes of turmeric (Curcuma longa L.). Ind. Eng. Chem. Res. 2000, 39, 4729-4733. [CrossRef]

65. Ling, J.; Wei, B.; Lv, G.; Ji, H.; Li, S. Anti-hyperlipidaemic and antioxidant effects of turmeric oil in hyperlipidaemic rats. Food Chem. 2012, 130, 229-235. [CrossRef]

(C) 2019 by the authors. Licensee MDPI, Basel, Switzerland. This article is an open access article distributed under the terms and conditions of the Creative Commons Attribution (CC BY) license (http://creativecommons.org/licenses/by/4.0/). 